\title{
Apparent motion display
}

\author{
ROBERT ADAMSON \\ Florida Atlantic University, Boca Raton, Florida 33432
}

\begin{abstract}
A control circuit is described which drives recurrently alternating projection tachistoscope shutters or lights for the study of apparent motion. The device, easily constructed, provides reliable variation in temporal duration of stimuli and the interval between their presentation.
\end{abstract}

Study of apparent motion requires control of stimuli intensity, control of stimulus size and/or distance from the viewer as well as interstimulus distance, and precise control of stimulus and interval duration. The use of viewing tachistoscopes, while affording reasonable control of duration. severely limits variation in viewing and visual angle. size. distance, etc. The equipment described herein affords a relatively unlimited variation of all such factors and allows the viewer to control interstimulus duration, thus enabling the average-error method to be employed.

The apparatus we have constructed consists of two 35-mn projectors (Kodak Carousel AF-AV-3402H) which rear-project separate images onto a $25 \times 122 \mathrm{~cm}$ surface. The screen can be variously placed between the projectors and the viewer, thus controlling viewing distance and angle. Control of image intensity is afforded by variable neutral-density filters placed in front of the projector lens. Electronic shutters (Gerbrands G1166), activated by a separate control circuit. control display duration for each image.

The projectors are table mounted and may be moved laterally, so that overlapping projection fields is possible. By composing slides with stimulus objects displaced to the central projection edge. it is possible to present a single bright visual field with minimal keystone effects. Alternatively, reversal slides (white or black) may be used for wide dark field presentation.

Figure 1 shows the electronic control for stimulus and interval timing. It is designed to turn on stimuli in continuous alternation for as long as the sircuit is activated. The stimuli shown are lights instead of the shutters described above, the simpler configuration being suitable for demonstrations of apparent motion.

The lights are turned on by one-shots (OS and OS2), which are precalibrated, by a method to be described, for desired stimulus du rations. For shutter control, the OS outputs activate reed relays, which in turn open the shutters. At the end of the stimulus interval, either OS1 or OS2 times out, delivering an off-pulse ( 0 to $-12 \mathrm{~V})$. Because of the characteristics of the modules used. these pulses cannot directly feed an OR-gate (OG), but

All components are BRS/LVE modules: OS 204, OG 204. FF 208. RD 206.

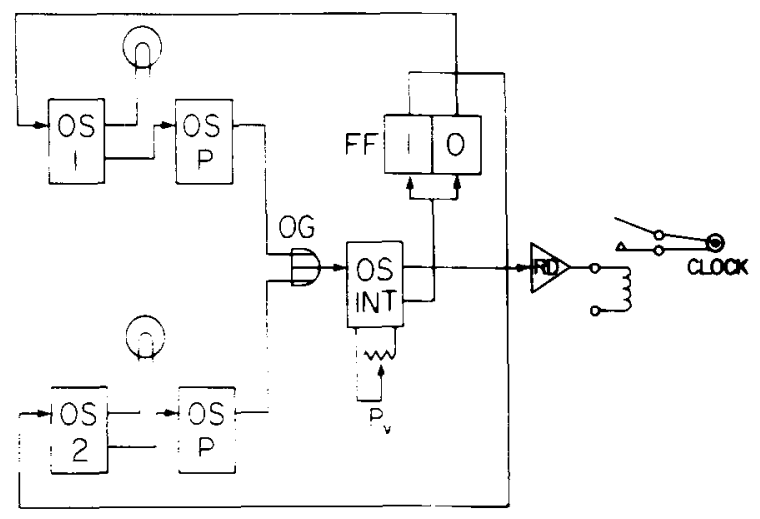

Figure 1. Control circuit for stimulus alternation in apparent motion.

must generate positive-going pulses $(-12$ to $0 \mathrm{~V}$ ) through $O S_{p}$. OS $\mathrm{p}$ provides a pulse of approximately 8 nsec with no external capacitance; other OS have 1.0 MF capacitance. The off-pulse of either stimulus control OS thus activates the interval control ( $\left.O S_{\text {int }}\right)$ through the OG.

The on-pulse of $O S_{\text {int }}$ drives a relay driver $(R D)$ and a stop clock (Lafayette Instrument Company digital clock, 54417) used for interval calibration. The off-pulse following the interval switches a flip-flop (FF) from its prior state.

When FF switches to a " 0 " condition, it generates a positive-going pulse to ${ } S_{1}$; when switched to a " 1 " condition. it pulses $\mathrm{OS}_{2}$. Consequently, its output alternates from one OS to the other following each interval. providing continuous oscillation between stimuli. The temporal succession is shown in Figure 2.

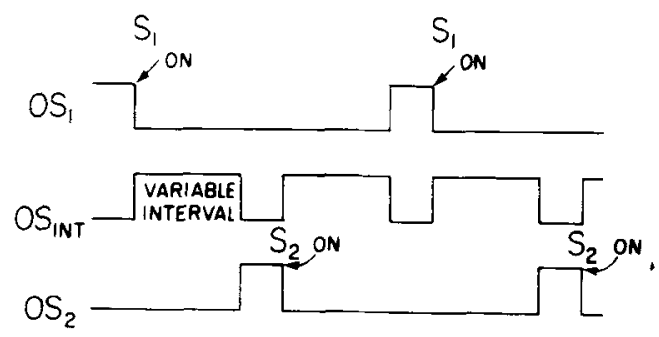

Figure 2. Timing diagram: $S_{1}$ and $S_{2}$ refer to stimuli controlled by $O S_{1}$ and $\mathrm{OS}_{2}$. 
Using a 1-MF Allied $188 \mathrm{P}$ capacitor with a $500-\mathrm{K}$ Bourns $3400 S$ pot. 100 trials were run at each of three settings: $0,250 \mathrm{~K}$, and $500 \mathrm{~K}$ ohms. The respective readings in milliseconds were: $14.9 \pm 1.5,321 \pm 1.2$, and $630.3 \pm 2$. Use of a $1 \%$ decade capacitor (General Radio 1419A) improved clock reliability to $\pm 1 \mathrm{msec}$, negligible in view of the additional expense involved. With additional capacitance (to $100 \mathrm{MF}$ ), it is possible to extend the timing range to approximately $30 \mathrm{sec}$.

$O S_{1}$ and $O S_{2}$ are precalibrated by the above method so that dial settings on the potentiometer correspond to desired stimulus durations. For average-error studies, $P_{V}$ is controlled by the viewer until he sees motion. The resultant dial set is then converted into interval time.

The one-shots, as modified, provide excellent sequential timers for other purposes. They may be linked in series with the off-pulse of the first OS furnishing input to the second, and so on.

(Received for publication September 9. 1975; revision received October 23. 1975.) 